Differences were apparent between the two hospices in the frequency of referrals to SLT. Staff had skills in caring for patients with communication impairments but some spoke of a desire for further training and closer links to SLT.

Conclusions Findings from this study and the literature suggest limited specialist communication support for patients accessing palliative care. Clinicians are encouraged to reflect upon the approach in their own setting to consider if changes could be implemented.

\section{P-117 IMPLEMENTING A HOSPICE TRAINING PROGRAMME FOR THE MANAGEMENT OF PATIENTS WITH AN ALTERED AIRWAY}

Joanne Reynolds, Liz Reed. Princess Alice Hospice, Esher, UK

\subsection{6/bmjspcare-2019-HUKNC.140}

Background Only 40\% of hospitals demonstrated good practice in the care of patients with tracheostomies. Six recommendations have been made to improve the care they receive. These include having a protocol and mandatory training to ensure that staff are competent in recognising and managing common airway complications (National Confidential Enquiry into Patient Outcome and Death, 2014). There is little research or evidence about the quality of care patients with an altered airway receive in adult hospices.

These patients usually have complex care needs and present a significant challenge for hospices in that staff are not caring for patients with an altered airway regularly enough to maintain competence and confidence in this area.

Aims In-patient (IPU) clinical staff will be competent and confident caring for patients with altered airways.

Intervention Mandatory, continuous education programme titled 'Essentials in tracheostomy and laryngectomy care in the hospice setting'.

Methods

- Pre- and post- education intervention on-line survey to all clinical staff undertaking the educational intervention;

- Practical simulation of the care of patients with an altered airway;

- Assessment of confidence and competence in caring for patients with an altered airway.

Findings This educational intervention is on-going. Analysis of the accumulative data will be undertaken in September 2019 and reported in the proposed paper.

Conclusion This appears to be the first evaluation of its kind; addressing retention of knowledge and assessment of competence/confidence in an adult hospice caring for patients with an altered airway.

\section{P-118 INVESTIGATING ANAEMIA AND TARGETING TREATMENT IN A HOSPICE POPULATION: A QUALITY IMPROVEMENT PROJECT}

Tom Steele, Helen Bonwick, Laura Chapman. Marie Curie Liverpool, Liverpool, UK

10.1136/bmispcare-2019-HUKNC.141

Background Anaemia is common in palliative care. Results of the national audit of blood transfusion practice in hospices, published (online first) last year, recommended more rigorous investigation of anaemia and increased use of alternative therapies (Neoh, Gray, Grant-Casey, Estcourt et al., 2019). This project aimed to improve the proportion of patients with anaemia at Marie Curie Hospice Liverpool having haematinic investigations and receiving appropriate, targeted therapies.

Methods Several changes have been implemented through this project. To raise awareness, education sessions were delivered and practical written guidance on performing and interpreting haematinic investigations disseminated. A protocol was implemented to ensure outpatients attending for blood transfusions had investigations completed. Finally, an electronic template was developed to prompt investigating and recording results. Between August 2018 and February 2019 continual retrospective analysis was performed using laboratory and patient records. All patients who had haemoglobin checked at the hospice had clinical details, haematinic tests (ferritin, iron studies, B12 and folate; if performed within 6 weeks before or 2 weeks after) and resulting treatments recorded.

Results 125 patients with anaemia were assessed over the project (excluding repeat sampling). Amongst those having blood transfusion, assessment of both iron status and B12/folate improved from $2 / 12(17 \%)$ in the first two months to $7 / 7$ $(100 \%)$ in the last two months of the project. Including all patients with $\mathrm{Hb}<100 \mathrm{~g} / \mathrm{dL}$, iron status assessment improved from $7 / 22(32 \%)$ to $12 / 18(67 \%)$ and B12/folate from $5 / 22$ $(23 \%)$ to $9 / 18$ (50\%). Results led to alternative therapies in 13 cases: oral iron $(n=5)$, intravenous iron $(n=6)$ and folic acid $(n=2)$.

Conclusions This quality improvement project has led to more patients being investigated for underlying causes of anaemia and increased usage of targeted therapies. Research is required to establish if this approach impacts symptoms or transfusion utilisation and the role of specific treatments, particularly intravenous iron therapy, in this population.

\section{P-119 THE EMOTIONAL IMPACT OF CANCER CACHEXIA: ARE HEALTHCARE PROFESSIONALS PROVIDING ADEQUATE SUPPORT?}

Sophie Rose Wood. St Helena, Colchester, UK

\subsection{6/bmjspcare-2019-HUKNC.142}

Background There remains no cure for cachexia. In the UK up to $60 \%$ of patients with a diagnosis of advanced cancer will develop cachexia. Palliative patients and their family frequently experience emotional distress as a result.

Aim To critically appraise the literature relating to the emotional impact of cachexia to make recommendation to change practice.

Methods A systematic literature review was conducted using five databases exploring literature from 2008 to 2018. The research question was structured using the PEO framework, which assisted in narrowing down pertinent keywords. Critical appraisal of the articles was conducted using tools such as the Critical Appraisal Skills Programme.

Results 1042 articles were identified, limits were applied followed by set inclusion and exclusion criteria narrowing the search to 29 articles. Following critical appraisal eight papers were included.

Conclusion Palliative patients and their family frequently experience emotional distress, as a result of cancer cachexia. The review identified the main factors that contribute or exacerbate the wide range of negative emotions experienced between 